\title{
Identification of the chloroplast adenosine-to-inosine tRNA editing enzyme
}

\author{
DANIEL KARCHER and RALPH BOCK
}

Max-Planck-Institut für Molekulare Pflanzenphysiologie, D-14476 Potsdam-Golm, Germany

\begin{abstract}
Plastids (chloroplasts) of higher plants exhibit two types of conversional RNA editing: cytidine-to-uridine editing in mRNAs and adenosine-to-inosine editing in at least one plastid genome-encoded tRNA, the tRNA-Arg(ACG). The enzymes catalyzing RNA editing reactions in plastids are unknown. Here we report the identification of the A-to-I tRNA editing enzyme from chloroplasts of the model plant Arabidopsis thaliana. The protein (AtTadA) has an unusual structure in that it harbors a large N-terminal domain of $>\mathbf{1 0 0 0}$ amino acids, which is not required for catalytic activity. The C-terminal region of the protein displays sequence similarity to tadA, the tRNA adenosine deaminase from Escherichia coli. We show that AtTadA is imported into chloroplasts in vivo and demonstrate that the in vitro translated protein triggers A-to-I editing in the anticodon of the plastid tRNA-Arg(ACG). Suppression of AtTadA gene expression in transgenic Arabidopsis plants by RNAi results in reduced A-to-I editing in the chloroplast tRNA-Arg(ACG). The RNAi lines display a mild growth phenotype, presumably due to reduced chloroplast translational efficiency upon limited availability of edited tRNA-Arg(ACG).
\end{abstract}

Keywords: Arabidopsis thaliana; plastid; chloroplast; TadA; RNA editing; inosine; adenosine deaminase; tRNA-Arg

\section{INTRODUCTION}

RNA editing is a post-transcriptional process changing the identity of individual nucleotides in primary transcripts. In higher plants, editing by pyrimidine transitions occurs in mRNAs of both DNA-containing cell organelles, the plastids (chloroplasts) and the mitochondria. mRNA editing in plastids of higher plants proceeds exclusively by cytidine-to-uridine (C-to-U) transitions (for review, see, e.g., Bock 2000, 2001; Schmitz-Linneweber and Barkan 2007). Direct in vivo evidence for the functional importance of plastid mRNA editing was obtained by the production of transplastomic (chloroplast-transformed) plants harboring a noneditable version of a plastid photosynthesis gene (Bock et al. 1994). The molecular recognition of mRNA editing sites is mediated by a cis-acting element residing in the region immediately upstream of the editing site (Bock et al. 1996, 1997; Chaudhuri and Maliga 1996; Hermann and Bock 1999; Miyamoto et al. 2002) and trans-acting protein factor(s) binding to the cis-element

Reprint requests to: Ralph Bock, Max-Planck-Institut für Molekulare Pflanzenphysiologie, Am Mühlenberg 1, D-14476 Potsdam-Golm, Germany; e-mail: rbock@mpimp-golm.mpg.de; fax: +49-331-567-8701.

Article published online ahead of print. Article and publication date are at http://www.rnajournal.org/cgi/doi/10.1261/rna.1600609.
(Chaudhuri et al. 1995; Bock and Koop 1997; Hirose and Sugiura 2001). Recently, members of the pentatricopeptide repeat (PPR) protein family have been identified as putative trans-factors involved in the specific recognition of mRNA editing sites in plastids (Kotera et al. 2005; Okuda et al. 2006; Chateigner-Boutin et al. 2008).

In addition to mRNA editing by $\mathrm{C}$-to- $\mathrm{U}$ conversions, plastids also carry out adenosine-to-inosine (A-to-I) editing. A-to-I editing occurs in at least one plastid genomeencoded tRNA, the tRNA-Arg(ACG) (Pfitzinger et al. 1990). The tRNA-Arg(ACG) is also edited in Escherichia coli and other eubacteria (Wolf et al. 2002). The editing event changes the adenosine in the wobble position (position 34) of the ACG anticodon to inosine. In E. coli and probably also in plastids, tRNA-Arg(ACG) is the only tRNA with inosine, whereas in eukaryotes, several tRNAs contain inosine in the wobble position of the anticodon. Evolutionary conservation of A-to-I editing in a single tRNA species, the tRNA-Arg(ACG), in E. coli and chloroplasts suggests that eubacteria and plastids use similar wobble rules for reading the (standard) genetic code.

In addition to A-to-I tRNA editing, eukaryotes also conduct mRNA editing by A-to-I conversions. These editing reactions occur in double-stranded regions of various mRNAs and are catalyzed by a family of enzymes termed adenosine deaminases acting on RNA (ADARs). 


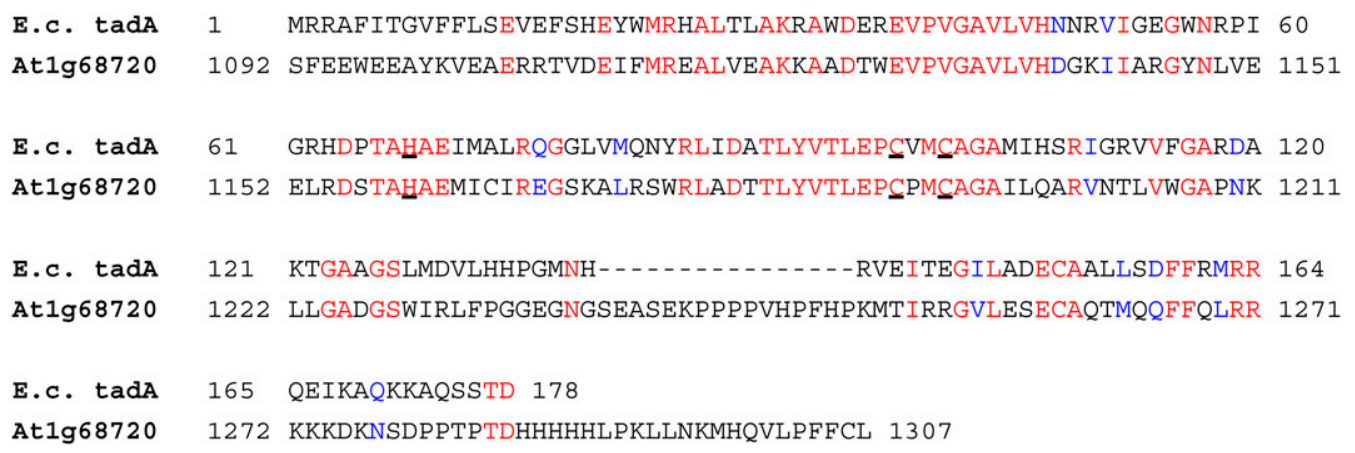

FIGURE 1. Partial sequence alignment of the tadA protein from Escherichia coli (E.c. tadA) and the candidate AtTadA protein from Arabidopsis thaliana (Atlg68720). Identical amino acid residues are shown in red, similar amino acid residues in blue. Amino acids involved in zinc binding are underlined. Note that Atlg68720 has a large N-terminal extension of $>1000$ amino acids, which has no sequence similarity to any protein of known function and is not shown here.

ADAR proteins consist of two or three dsRNA-binding domains and a catalytic deaminase domain (Polson and Bass 1994; O'Connell and Keller 1994; Hurst et al. 1995; O'Connell et al. 1995; Dabiri et al. 1996; Melcher et al. 1996). The latter comprises three zinc-binding amino acid residues (Fig. 1; Rubio et al. 2007) and a proton-shuttling glutamate (Bass 1997; Gerber and Keller 2001; Keegan et al. 2001). The catalytic domain of eukaryotic wobble-specific A-to-I tRNA deaminases has a similar structure (Maas et al. 1999; Rubio et al. 2007), and these editing enzymes have been termed ADATs (adenosine deaminases acting on tRNA). Based on sequence similarity with the catalytic subunit of a yeast ADAT enzyme, the E. coli protein that conducts the single A-to-I editing event in tRNA$\operatorname{Arg}(A C G)$ was identified (Wolf et al. 2002). The bacterial enzyme was termed tRNA-specific adenosine deaminase ( $\operatorname{tadA})$, and is encoded by an essential gene in E. coli. Essentiality of A-to-I editing in tRNA-Arg(ACG) is most probably due to the requirement for inosine in the wobble position to decode the three arginine codons CGU, CGC, and CGA (Crick 1966). tadA forms a homodimer and, in addition to the E. coli tRNA-Arg(ACG), the enzyme can also edit the yeast tRNA-Arg (Wolf et al. 2002). The anticodon stem-loop of the tRNA molecule was shown to harbor the essential substrate recognition elements for sitespecific A-to-I editing via the hydrolytic deamination reaction catalyzed by tadA (Wolf et al. 2002).

In chloroplasts, neither the identity of the C-to-U mRNA editing enzyme nor the identity of the A-to-I tRNA editing enzyme have been revealed. As all known cytidine deaminases and adenosine deaminases involved in conversional RNA editing display a similar structural organization of their catalytic deaminase domains (Gerber and Keller 2001; Keegan et al. 2001; Rubio et al. 2007), we performed a comprehensive search for candidate deaminases acting on polynucleotides in the fully sequenced genome of the model plant Arabidopsis thaliana. We report here the identification of the chloroplast A-to-I tRNA editing enzyme.

\section{RESULTS AND DISCUSSION}

\section{A chloroplast-targeted Arabidopsis protein with local sequence similarity to tadA}

Using the consensus sequence motifs in the catalytic domain of editing deaminases (Rubio et al. 2007), we performed a Patmatch search (http://www.arabidopsis.org/ cgi-bin/patmatch/nph-patmatch.pl) of the fully sequenced genome of the model plant Arabidopsis thaliana (The Arabidopsis Genome Initiative 2000) to identify candidate editing deaminases. Candidate proteins were further analyzed by BLAST (http://blast.ncbi.nlm.nih.gov/Blast.cgi) searches to identify potential homologs from other species. In the course of these analyses, a large open reading frame (Arabidopsis thaliana gene number At1g68720) was identified, whose $\mathrm{C}$ terminus displayed sequence similarity with the Escherichia coli tRNA editing deaminase tadA (Fig. 1). While the E. coli tadA is a small protein of only 178 amino acids, the Atlg68720 reading frame has 1307 amino acids and the sequence homology with $\operatorname{tad} \mathrm{A}$ is confined to amino acid residues 1106-1285 (Fig. 1). The large additional $\mathrm{N}$-terminal sequence of $>1000$ amino acids does not display any significant similarity to any protein domain of known function.

To test if Atlg68720 encodes an organellar editing deaminase, we first wanted to determine the subcellular localization of the protein. To this end, we translationally fused the sequence encoding the N-terminal 177 amino acids of Atlg68720 to the gene for the green fluorescence protein (GFP). Transit peptides for protein import into plant cell organelles reside in the $\mathrm{N}$ terminus and are usually shorter than 100 amino acids (Heazlewood et al. 2005; Lee et al. 2008). The At1g68720-GFP fusion gene was inserted into a plant expression vector and transformed into isolated protoplasts by polyethylene glycol-mediated DNA uptake. Analysis of GFP fluorescence in transformed protoplasts revealed strong fluorescence that localized 

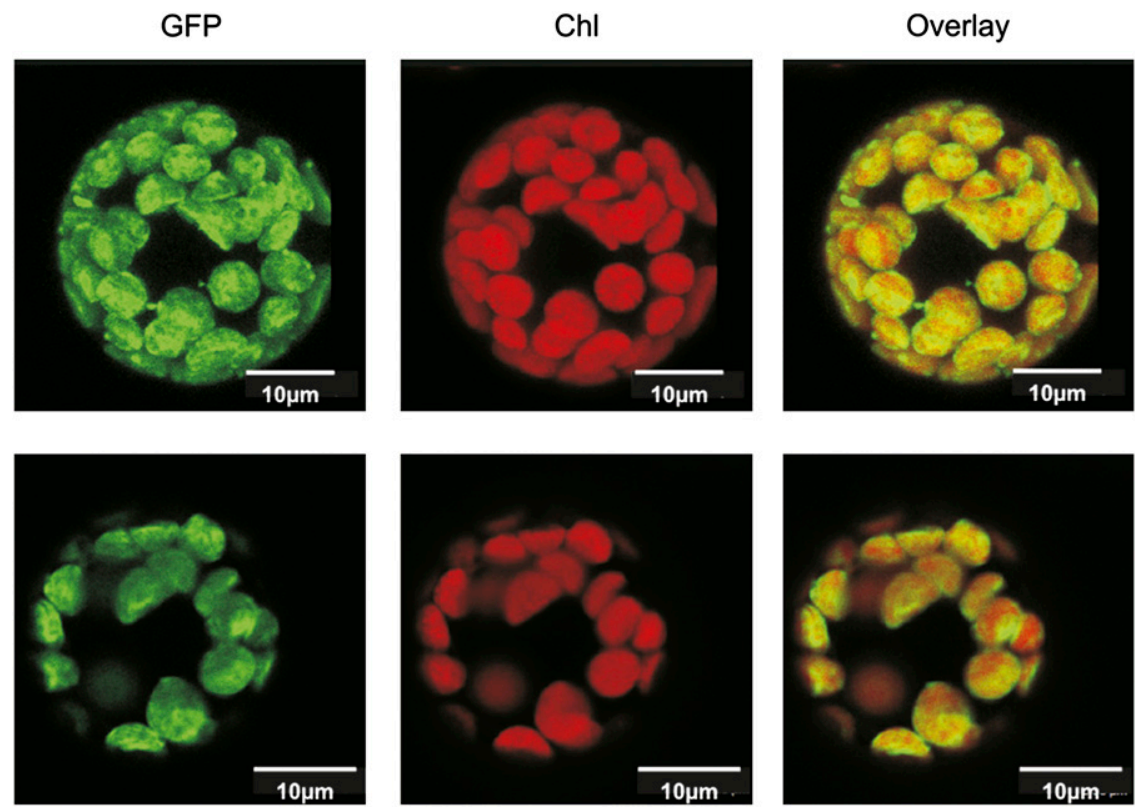

FIGURE 2. Analysis of subcellular localization of the candidate AtTadA protein from Arabidopsis thaliana. A chimeric protein consisting of the first 177 amino acids of Atlg68720 fused to GFP is targeted to chloroplasts in transformed tobacco protoplasts. Fluorescence of the green fluorescence protein (GFP), chlorophyll fluorescence (Chl), and the overlay of the two fluorescences are shown for two transformed protoplasts.

exclusively to chloroplasts (Fig. 2). This suggests that the Atlg68720 protein is targeted to plastids, and makes it a candidate chloroplast editing deaminase.

\section{RNAi suppression of At1g68720 reduces A-to-I editing in tRNA-Arg(ACG)}

In order to test whether the Atlg68720 protein is indeed a plastid editing deaminase, we sought to address its function by reverse genetics. We first searched the collection of T-DNA insertion lines available at the Nottingham Arabidopsis Stock Center (http://arabidopsis.info/). We identified two independent T-DNA insertion lines associated with locus At1g68720. Unfortunately, none of the two lines harbored the T-DNA insertion within the At1g68720 coding region. In one of the lines, the T-DNA resided 135 base pairs (bp) upstream of the start codon of Atlg68720 and in the second line, the insertion site was even $560 \mathrm{bp}$ upstream of the start codon. As T-DNA insertions upstream of the reading frame sometimes inhibit gene expression by disrupting essential promoter or 5' UTR elements, we analyzed At1g68720 mRNA levels in homozygous plants of both mutants by real-time quantitative PCR. These experiments revealed that the At1g68720 transcript levels in the T-DNA lines are similar or even slightly higher than in the wild type (data not shown). (Slightly higher transcript levels can originate from readthrough transcription initiating within the strongly transcribed upstream T-DNA sequence.) This suggests that neither of the two T-DNA insertion lines represents an Atlg68720 mutant. Consistent with this interpretation, no defects in chloroplast mRNA or tRNA editing could be identified in homozygous T-DNA insertion lines (data not shown).

In the absence of T-DNA knock-out lines for Atlg68720, we next attempted to produce mutant plants by RNA interference (RNAi). Agrobacteriummediated transformation of Arabidopsis plants with a hairpin-type RNAi construct (Hilson et al. 2004) against At1g68720 yielded several independent transgenic lines, which were subsequently assayed for successful downregulation of Atlg68720 by real-time quantitative PCR. These analyses identified three lines (subsequently referred to as RNAi-tadA-E, RNAi-tadA-F, and RNAi-tadA-H), which showed strong suppression of At1g68720 expression (Fig. 3A). RNAi was strongest in line RNAi-tadA-F, with the residual expression level of Atlg68720 being $<10 \%$ of the expression in the wild type (Fig. 3A).

Having successfully isolate RNAi lines for the candidate plastid editing deaminase gene At1g68720, we next used the mutant plants to test for chloroplast RNA editing efficiency. Analysis of C-to-U mRNA editing at eight sites (six sites in the $n d h B$ transcript: in codons 249, 277, 279, 291, 419, and 494; and two sites in the rpoB transcript: in codons 113 and 184) revealed no difference in editing efficiency between the wild-type and the RNAi lines (data not shown), suggesting that At1g68720 most likely does not represent the C-to- $\mathrm{U}$ mRNA editing deaminase of the chloroplast. However, when we tested A-to-I editing in the anticodon of the plastid tRNA-Arg(ACG), all three RNAi lines showed drastically reduced editing efficiency (Fig. 3B). Importantly, the residual editing activity correlated well with the residual expression levels of At1g68720 in the RNAi lines, in that it was highest in line RNAi$\operatorname{tadA}-\mathrm{H}$ and lowest in line RNAi-tadA-F (Fig. 3, cf. A and B). This suggests that the Atlg68720 gene encodes the chloroplast A-to-I tRNA editing deaminase, and we therefore tentatively named the gene Arabidopsis thaliana tRNA adenosine deaminase A (AtTadA). This name should be unambiguous also with respect to the chloroplast localization of the enzyme, because (1) there is no inosine in plant mitochondrial tRNAs and (2) the yet to be identified nuclear A-to-I tRNA editing enzyme should be named AtADAT, in accordance with the nomenclature for eukaryotic adenosine deaminases acting on tRNA substrates. 
A

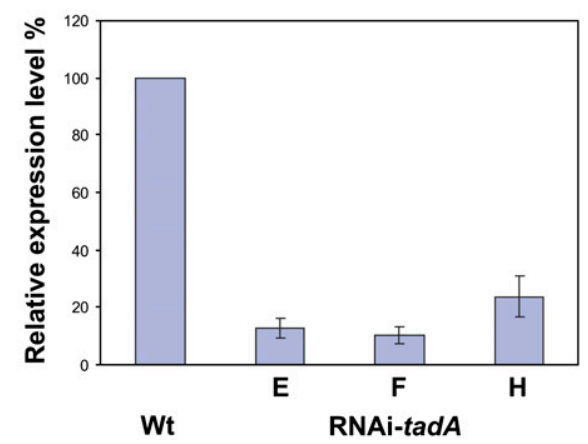

B
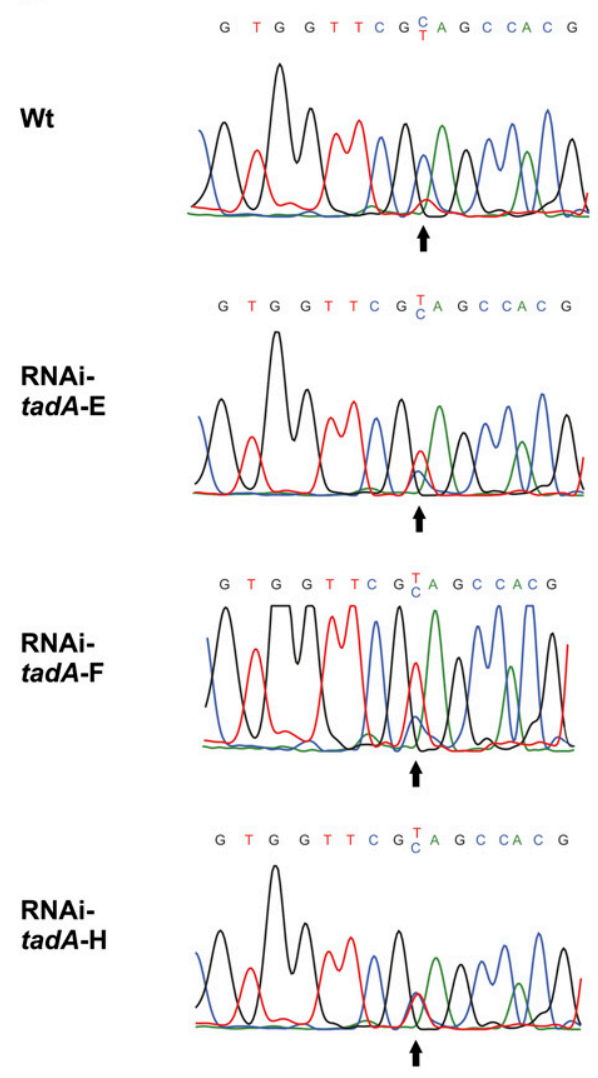

FIGURE 3. Analysis of tRNA editing in transgenic Arabidopsis plants harboring anRNAi construct targeted against the candidate AtTadA gene, Atlg68720. (A) Down-regulation of At1g68720 expression in three independently generated RNAi lines (RNAi-tadA-E, RNAi-tadA-F, and RNAi-tadA-H). RT-PCR data comprise three biological replicas. Error bars indicate standard deviation. (B) Analysis of A-to-I editing in the anticodon of the chloroplast genome-encoded tRNA$\operatorname{Arg}(\mathrm{ACG})$. Note that the sequence chromatographs show the sequence of the complementary strand and, therefore, A-to-I conversion is seen as T-to-C conversion here. While the wild type (Wt) shows nearly complete A-to-I editing (C peak marked by arrow), the RNAi lines show drastically reduced editing, the extent of which correlates with the level of downregulation of At1g68720 by RNAi $(A)$.

\section{Phenotype of AtTadA RNAi lines}

In view of the strongly reduced A-to-I editing in the anticodon of the plastid tRNA-Arg(ACG), we next wanted to determine the phenotypic consequences of AtTadA suppression in the RNAi lines. The ACG to ICG modification of the anticodon is believed to be required to read all four CGN codons for arginine (Pfitzinger et al. 1990; Sugiura and Sugita 2004). The tRNA with the unmodified anticodon should not be suitable to decode CGA and CGG triplets (Crick 1966). Thus, although a residual fraction of modified plastid tRNA-Arg(ACG) is still present even in the strongest RNAi lines, it seems reasonable to suspect that chloroplast translation should proceed somewhat less efficiently in the RNAi lines than in the wild type, due to the limited availability of tRNA molecules decoding CGA and CGG arginine triplets. Previous work in tobacco plants with mutated plastid tRNA genes has shown that a reduced wobbling efficiency in the codon-anticodon interaction results in reduced translational efficiency, which in turn impairs photosynthetic performance and plant growth (Rogalski et al. 2008). When we compared wild-type plants and AtTadA RNAi plants under different growth conditions, we observed only a mild growth phenotype in the strong RNAi lines (Fig. 4). Growth and development of the RNAi lines were somewhat delayed, and this phenotype was most pronounced under long-day conditions. The strength of the phenotype correlated well with both the strength of AtTadA down-regulation and the residual editing activity on tRNA-Arg(ACG) (cf. Figs. 3 and 4). The phenotype was not nearly as strong as in other plastid tRNA mutants (Rogalski et al. 2008), suggesting that the residual population of edited (inosine-containing) tRNA-Arg(ACG) molecules is sufficient to sustain fairly high translation rates in the chloroplast.

\section{In vitro editing of chloroplast tRNA-Arg(ACG) by the AtTadA protein}

In order to ultimately confirm that the AtTadA protein has A-to-I tRNA editing activity, we wanted to test whether the overexpressed protein is capable of editing the tRNA$\operatorname{Arg}(\mathrm{ACG})$ in vitro. To this end, we amplified the cDNA portion of Atlg68720 that is homologous to the E. coli tadA protein (amino acids 1103-1307) (Fig. 1) and produced the corresponding protein by in vitro translation. We then added RNA extracted from an RNAi-tadA-F plant, the RNAi line with the lowest level of edited tRNA-Arg(ACG) (Fig. 3). As a control, GFP was produced by in vitro translation and also incubated with RNA from the RNAitadA-F line. A significant conversion of unedited into edited tRNA-Arg(ACG) was observed already after 5-min incubation of RNAi-tadA-F RNA with the in vitro translated AtTadA protein (Fig. 5). A-to-I conversion in the anticodon was nearly complete after 15-min incubation, 

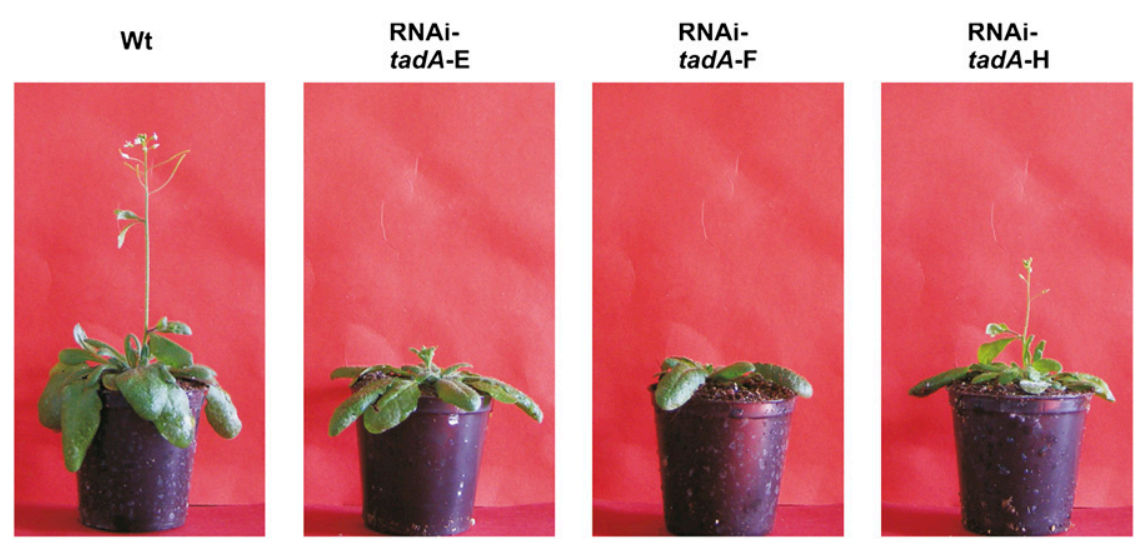

The insertion in line N595878 lies 560 bp upstream of the start codon of Atlg68720. Homozygous T-DNA insertion plants were identified by PCR using genomic DNA and the T-DNA left border-specific primer LbXL (5'-CCGATTTCGGAACCACCATCAAACA GG-3') in combination with RP-N665576 (5'-AACCCCATAAAGCACAAATCCA-3') or RP-N595878 (5'-GTTCGAGATTAAG ATTGGGCC-3'). Wild-type alleles were identified by PCR using the RP primer in combination with LP primers (LP-N6655 76: 5'-GCCCTTTGGCATAGAAATGG-3' or LP-N595878: 5'-GAAGATCCATGTTATCT CGCC- $3^{\prime}$ ).

FIGURE 4. Phenotype of RNAi-tadA plants. Wild-type (Wt) and mutant plants were grown under long-day conditions at a light intensity of $180 \mu \mathrm{E} \mathrm{m}^{-2} \mathrm{sec}^{-1}$. All RNAi lines show a mild growth retardation as evidenced by delayed flowering. The delayed growth correlates with the level of down-regulation of Atlg68720 and the concomitant reduction in tRNA editing efficiency in the individual RNAi lines (Fig. 3).

\section{Plant transformation and isolation of RNAi lines}

The transformation vector used for downregulation of the Atlg68720 gene was plas-

whereas no significant editing occurred in the presence of in vitro translated GFP protein (Fig. 5). These data strongly suggest that the AtTadA protein has indeed A-to-I tRNA editing activity. Moreover, they demonstrate that the C-terminal domain of the AtTadA protein (Fig. 1) is sufficient to conduct the editing reaction, at least in vitro. At present, we can only speculate about the function of the large N-terminal extension of the AtTadA protein compared with the E. coli tadA protein (Fig. 1). This extension is conserved in the homologous proteins from rice (Oryza sativa), poplar (Populus trichocarpa), and castor bean (Ricinus communis), suggesting that it serves some important function in vivo. Whether it regulates enzyme activity and/or substrate specificity, or serves some other function, remains to be determined.

In summary, our data strongly suggest that the Atlg68720 gene encodes the chloroplast A-to-I tRNA editing deaminase. With this, the first RNA editing enzyme from plant organelles is now identified and available for detailed structural and functional analysis. The C-to-U editing enzymes from chloroplasts and plant mitochondria remain elusive and still await their molecular identification.

\section{MATERIALS AND METHODS}

\section{Plant material}

Arabidopsis thaliana wild-type and mutant plants (ecotype Col-0) were grown under standard glasshouse conditions. Phenotypic assays were performed under long-day conditions (in a 16-h light/ 8 -h dark regime) at a light intensity of $180 \mu \mathrm{E} \mathrm{m}^{-2} \mathrm{sec}^{-1}$. Two independent T-DNA insertion lines associated with locus At1g68720 were obtained from the Nottingham Arabidopsis Stock Center (http://arabidopsis.info/). The insertion in line N665576 is $135 \mathrm{bp}$ upstream of the start codon (6 bp upstream of exon 1). mid CATMA1a58100 from the AGRIKOLA collection of RNAi vectors (Hilson et al. 2004). The transformation vector harbored a 401-bp PCR product covering the region from nucleotide 3014 to 3414 of the At1g68720 coding region. The plasmid was transformed into Agrobacterium tumefaciens strain GV3101::pMP90::pSOUP. Arabidopsis thaliana Col-0 plants were transformed by the floral dip method (Clough and Bent 1998). Transgenic plants were selected for BASTA resistance by spraying of seedlings with phosphinotricine (Duchefa) at a concentration of $40 \mu \mathrm{g} / \mathrm{mL}$ five times at 2-d intervals.

\section{Isolation of nucleic acids}

Total plant DNA was extracted from fresh leaf tissue samples by a cetyltrimethylammoniumbromide (CTAB)-based method (Doyle and Doyle 1990). Total cellular RNA was isolated with the TriFast reagent (Peqlab $\mathrm{GmbH}$ ) following the manufacturer's protocol. For synthesis of cDNA, RNA samples were treated with DNase I (Epicentre Biotechnologies) to eliminate residual contaminating genomic DNA. tRNA was purified by separating $10 \mu \mathrm{g}$ of total RNA (extracted from wild-type plants or RNAi lines) on ureacontaining $8 \%$ polyacrylamide gels followed by staining with ethidiumbromide. The tRNA fraction was excised from the gel and the excised gel slices were incubated in $200 \mu \mathrm{L} 0.3 \mathrm{M} \mathrm{NaCl}$ overnight at $4^{\circ} \mathrm{C}$ under continuous shaking. After addition of 0.1 vol $3 \mathrm{M}$ sodium acetate, the eluted tRNA was precipitated with 2.5 vol ethanol.

\section{cDNA synthesis, PCR, and DNA sequencing}

RNA samples treated with RNase-free DNase I were reverse transcribed with SuperScript III reverse transcriptase (Invitrogen) using oligo- $\mathrm{dT}_{18}$ primer and following the instructions of the supplier. cDNAs were used as templates for quantitative real-time PCR with gene-specific primers. The primers for the Atlg68720 gene were Pq1g68720for (5'-CGAGCTGGAAGCCCTGACT-3') and Pq1g68720rev (5'-TGATTCGTTGGGTGAACTCCTT-3'). Real-time PCR was performed with the StepOnePlus Real-Time PCR System (Applied Biosystems) using Absolute SYBR Green 
ROX Mix (Thermo Scientific). UBQ10 (At4g05320) and the Tipp41-like family protein (At4G34270) were detected in parallel and used as internal controls. The primer pair used for amplification of At4g05320 was PubiFw (5'-GGCCTTGTATAATCCCT GATGAATAAG-3') and PubiRev (5'-AAAGAGATAACAGGAA CGGAAACATAGT-3'), the primers for At4G34270 were PTipp41for $\left(5^{\prime}\right.$-TCAACTGGATACCCTTTCGCA-3') and PTipp41rev (5'-GTGAAAACTGTTGGAGAGAAGCAA-3'). Three biological and three technical replicates were analyzed. The $2^{-\Delta \Delta C T}$ method was used to determine the relative transcript levels (Livak and Schmittgen 2001).

To analyze the editing status of tRNA-Arg(ACG), purified tRNAs were ligated to oligonucleotide P3' Lig ( $5^{\prime}$-rCrCTGCAGC TACTGGCCGTCGTTTTACTCrAox-3') using T4 RNA ligase (New England Biolabs). This oligonucleotide carries two ribonucleotides at its $5^{\prime}$ end as well as a ribonucleotide with an oxidized ribose moiety (dialdehyde) at its $3^{\prime}$ end to prevent self-ligation. cDNA synthesis was performed using oligonucleotide PM13 (GTAAAACGACGGCCAGT) as a primer, which is complementary to P3' Lig. For amplification of tRNA-Arg(ACG), oligonucleotides PcpArg-F (GGGCTTGTAGCTCAGAGG), and PM13tRNA (GGCCAGTAGCTGCAGGTGG) were used. Primer PM13tRNA ends with TGG and thus is specific for mature tRNAs carrying the post-transcriptionally added CCA trinucleotide at their $3^{\prime}$ ends. Purified PCR products were sequenced with primer PM13tRNA.

\section{Analysis of subcellular localization}

The first $399 \mathrm{bp}$ of the coding sequence of Atlg68720 were amplified using Phusion DNA polymerase (Finnzymes) and the gene-specific primers P1g68720for (5'-TTCTCGAGATGT TCAATACATACACAAAC- $\left.3^{\prime}\right)$ and P1g68720rev (5'-TTCCA TGGCACAACATTCATCAGATTC-3') followed by digestion with the restriction enzymes XhoI and NcoI. The restriction sites had been introduced into the PCR product with the primer sequences (recognition sequences underlined). The DNA fragment was cloned into the respective restriction sites of the vector pA7-GFP (Voelker et al. 2006) to generate a translational fusion between the $\mathrm{N}$ terminus of Atg68720 and the coding sequence for the GFP. Preparation and transient transformation of tobacco (Nicotiana tabacum) protoplasts was done according to published protocols (Huang et al. 2002). Subcellular localization of GFP fluorescence was determined by confocal laser-scanning microscopy (TCS SP2; Leica) using an argon laser for excitation (at $488 \mathrm{~nm}$ ), a $500-510-\mathrm{nm}$ filter for detection of GFP fluorescence, and a 610-700-nm filter for detection of chlorophyll fluorescence.
$0 \min$
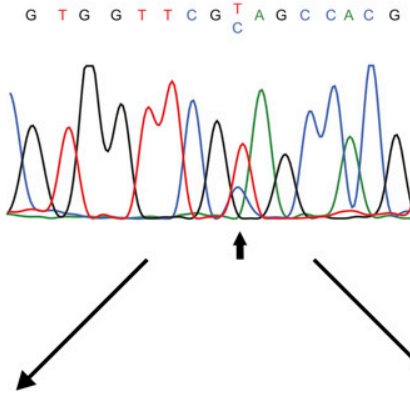

GFP

$5 \min$

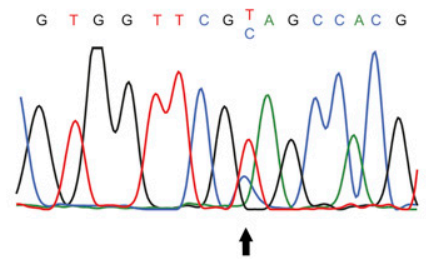

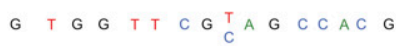

$15 \min$

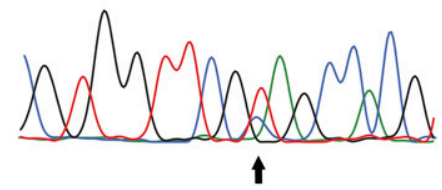

TadA
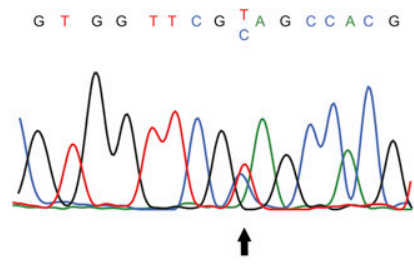

$G \quad T G G T T C G_{T}^{C} A G C C A C G$

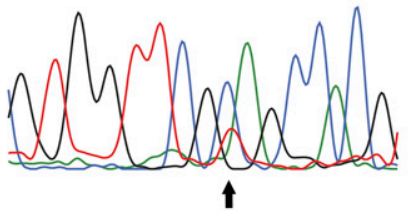

FIGURE 5. A-to-I RNA editing activity of the chloroplast AtTadA protein in vitro. Largely unedited tRNA-Arg(ACG) from an RNAi-tadA-F plant was used as substrate for in vitro editing assays with the chloroplast AtTadA protein produced by in vitro translation. As a control, GFP was produced by in vitro translation. While incubation with the AtTadA protein leads to time-dependent A-to-I conversion in the anticodon of tRNA-Arg(ACG), the tRNA remains largely unedited in the GFP control. The chromatographs show the sequence of the complementary strand. A-to-I conversion is, therefore, seen as T-to-C conversion here.

\section{In vitro expression of AtTadA and enzyme activity assay}

The cDNA portion of Atlg68720 that is homologous to the E. coli tadA protein (amino acids 1103-1307) (Fig. 1) was amplified using Phusion DNA polymerase (Finnzymes) and the oligonucleotide primers PAtTadA205for (5'-CTTTAAGAAGGAGATATACCATGGAA GCTGAAAGACGAACAG-3') and PAtTadArev (5' -TGATGATGAGAACCCCCCCCTTACA GACAGAAAAAGGGTAAG-3'). The PCR product was used as a template to add the T7 promoter and terminator sequences with the RTS E. coli Linear Template Generation Set (Roche Applied Science) according to the manufacturer's protocol. In vitro translation was performed with the RTS 100 E. coli HY Kit (Roche Applied Science) according to the manufacturer's instruction. The AtTadA was synthesized from $100 \mathrm{ng}$ of linear PCR product. As a control, GFP was produced by in vitro translation with $1 \mu \mathrm{g}$ of the GFP control vector provided by the manufacturer (Roche Applied Science). To test for A-to-I editing activity on tRNA-Arg(ACG), $3 \mu \mathrm{L}$ of the AtTadA in vitro translation reaction were incubated with $10 \mu \mathrm{g}$ total RNA extracted from the RNAi-tadA-F line for 5 or $15 \mathrm{~min}$ at $30^{\circ} \mathrm{C}$ in the reaction buffer described by Wolf et al. (2002). As a control, RNA was incubated with a $3 \mu \mathrm{L}$ aliquot of the GFP in vitro translation reaction. tRNA isolation, reverse transcription, and sequencing were done as described above. 


\section{ACKNOWLEDGMENTS}

We thank the MPI-MP Green Team for plant care and cultivation, Dr. Thomas Altmann and Astrid Basner for providing the AGRIKOLA clone, Armin Schlereth for help with real-time PCR analyses, and Anja Klevesath for excellent technical assistance. This research was supported by the Max Planck Society.

Received February 15, 2009; accepted April 6, 2009.

\section{REFERENCES}

Bass BL. 1997. RNA editing and hypermutation by adenosine deamination. Trends Biochem Sci 22: 157-162.

Bock R. 2000. Sense from nonsense: How the genetic information of chloroplasts is altered by RNA editing. Biochimie 82: 549-557.

Bock R. 2001. RNA editing in plant mitochondria and chloroplasts. In Frontiers in molecular biology: RNA editing (ed. B Bass), pp. 38-60. Oxford University Press, New York:

Bock R, Koop H-U. 1997. Extraplastidic site-specific factors mediate RNA editing in chloroplasts. EMBO J 16: 3282-3288.

Bock R, Kössel H, Maliga P. 1994. Introduction of a heterologous editing site into the tobacco plastid genome: The lack of RNA editing leads to a mutant phenotype. EMBO J 13: 4623-4628.

Bock R, Hermann M, Kössel H. 1996. In vivo dissection of cis-acting determinants for plastid RNA editing. EMBO J 15: 5052-5059.

Bock R, Hermann M, Fuchs M. 1997. Identification of critical nucleotide positions for plastid RNA editing site recognition. RNA 3: 1194-1200.

Chateigner-Boutin A-L, Ramos-Vega M, Guevara-García A, Andrés C, Gutiérrez-Nava M, Cantero A, Delannoy E, Jiménez LF, Lurin C, Small I, et al. 2008. CLB19, a pentatricopeptide repeat protein required for editing of rpoA and clpP chloroplast transcripts. Plant J 56: 590-602.

Chaudhuri S, Maliga P. 1996. Sequences directing C-to-U editing of the plastid psbL mRNA are located within a 22 nucleotide segment spanning the editing site. EMBO J 15: 5958-5964.

Chaudhuri S, Carrer H, Maliga P. 1995. Site-specific factor involved in the editing of the psbL mRNA in tobacco plastids. EMBO $J$ 14: 2951-2957.

Clough SJ, Bent AF. 1998. Floral dip: A simplified method for Agrobacterium-mediated transformation of Arabidopsis thaliana. Plant J 16: 735-743.

Crick FHC. 1966. Codon-anticodon pairing: The wobble hypothesis. J Mol Biol 19: 548-555.

Dabiri GA, Lai F, Drakas RA, Nishikura K. 1996. Editing of the GluR$B$ ion channel RNA in vitro by recombinant double-stranded RNA adenosine deaminase. EMBO J 15: 34-45.

Doyle JJ, Doyle JL. 1990. Isolation of plant DNA from fresh tissue. Focus 12: 13-15.

Gerber AP, Keller W. 2001. RNA editing by base deamination: More enzymes, more targets, new mysteries. Trends Biochem Sci 26: 376377.

Heazlewood JL, Tonti-Filippini J, Verboom RE, Millar AH. 2005. Combining experimental and predicted datasets for determination of the subcellular location of proteins in Arabidopsis. Plant Physiol 139: 598-609.

Hermann M, Bock R. 1999. Transfer of plastid RNA-editing activity to novel sites suggests a critical role for spacing in editing-site recognition. Proc Natl Acad Sci 96: 4856-4861.

Hilson P, Allemeersch J, Altmann T, Aubourg S, Avon A, Beynon J, Bhalerao RP, Bitton F, Caboche M, Cannoot B, et al. 2004. Versatile gene-specific sequence tags for Arabidopsis functional genomics: Transcript profiling and reverse genetics applications. Genome Res 14: 2176-2189.

Hirose T, Sugiura M. 2001. Involvement of a site-specific trans-acting factor and a common RNA-binding protein in the editing of chloroplast mRNAs: Development of a chloroplast in vitro RNA editing system. EMBO J 20: 1144-1152.

Huang F-C, Klaus SMJ, Herz S, Zou Z, Koop H-U, Golds TJ. 2002. Efficient plastid transformation in tobacco using the aphA-6 gene and kanamycin selection. Mol Genet Genomics 268: 19-27.

Hurst SR, Hough RF, Aruscavage PJ, Bass BL. 1995. Deamination of mammalian glutamate receptor RNA by Xenopus dsRNA adenosine deaminase: Similarities to in vivo RNA editing. RNA 1: 10511060.

Keegan LP, Gallo A, O'Connell MA. 2001. The many roles of an RNA editor. Nat Rev Genet 2: 869-878.

Kotera E, Tasaka M, Shikanai T. 2005. A pentatricopeptide repeat protein is essential for RNA editing in chloroplasts. Nature 433: 326-330.

Lee DW, Kim JK, Lee S, Choi S, Kim S, Hwang I. 2008. Arabidopsis nuclear-encoded plastid transit peptides contain multiple sequence subgroups with distinctive chloroplast-targeting sequence motifs. Plant Cell 20: 1603-1622.

Livak KJ, Schmittgen TD. 2001. Analysis of relative gene expression data using real-time quantitative PCR and the $2^{-\Delta \Delta \mathrm{C}(\mathrm{T})}$ Method. Methods 25: 402-408.

Maas S, Gerber AP, Rich A. 1999. Identification and characterization of a human tRNA-specific adenosine deaminase related to the ADAR family of per-mRNA editing enzymes. Proc Natl Acad Sci 96: $8895-8900$.

Melcher T, Maas S, Herb A, Sprengel R, Seeburg PH, Higuchi M. 1996. A mammalian RNA editing enzyme. Nature 379: 460-464.

Miyamoto T, Obokata J, Sugiura M. 2002. Recognition of RNA editing sites is directed by unique proteins in chloroplasts: Biomedical identification of cis-acting elements and trans-acting factors involved in RNA editing in tobacco and pea chloroplasts. Mol Cell Biol 22: 6726-6734.

O’Connell MA, Keller W. 1994. Purification and properties of doublestranded RNA-specific adenosine deaminase from calf thymus. Proc Natl Acad Sci 91: 10596-10600.

O’Connell MA, Krause S, Higuchi M, Hsuan JJ, Totty NF, Jenny A, Keller W. 1995. Cloning of cDNAs encoding mammalian doublestranded RNA-specific adenosine deaminase. Mol Cell Biol 15: 1389-1397.

Okuda K, Nakamura T, Sugita M, Shimizu T, Shikanai T. 2006. A pentatricopeptide repeat protein is a site recognition factor in chloroplast RNA editing. J Biol Chem 281: 37661-37667.

Pfitzinger H, Weil JH, Pillay DTN, Guillemaut P. 1990. Codon recognition mechanisms in plant chloroplasts. Plant Mol Biol 14: 805-814.

Polson AG, Bass BL. 1994. Preferential selection of adenosines for modification by double-stranded RNA adenosine deaminase. EMBO J 13: 5701-5711.

Rogalski M, Karcher D, Bock R. 2008. Superwobbling facilitates translation with reduced tRNA sets. Nat Struct Mol Biol 15: 192198.

Rubio MAT, Pastar I, Gaston KW, Ragone FL, Janzen CJ, Cross GAM, Papavasiliou FN, Alfonzo JD. 2007. An adenosine-to-inosine tRNA-editing enzyme that can perform C-to-U deamination of DNA. Proc Natl Acad Sci 104: 7821-7826.

Schmitz-Linneweber C, Barkan A. 2007. RNA splicing and RNA editing in chloroplasts. Top Curr Genet 19: 213-248.

Sugiura C, Sugita M. 2004. Plastid transformation reveals that moss tRNA $^{\text {Arg }}$-CCG is not essential for plastid function. Plant J 40: 314321.

The Arabidopsis Genome Initiative. 2000. Analysis of the genome sequence of the flowering plant Arabidopsis thaliana. Nature 408: 796-815.

Voelker C, Schmidt D, Mueller-Roeber B, Czempinski K. 2006. Members of the Arabidopsis AtTPK/KCO family form homomeric vacuolar channels in planta. Plant J 48: 296-306.

Wolf J, Gerber AP, Keller W. 2002. tadA, an essential tRNA-specific adenosine deaminase from Escherichia coli. EMBO J 21: 38413851. 

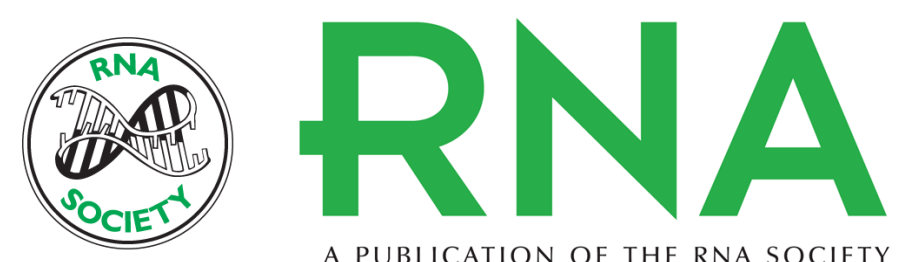

A PUBLICATION OF THE RNA SOCIETY

\section{Identification of the chloroplast adenosine-to-inosine tRNA editing enzyme}

Daniel Karcher and Ralph Bock

RNA 2009 15: 1251-1257 originally published online May 21, 2009

Access the most recent version at doi:10.1261/rna.1600609

$\begin{array}{ll}\text { References } & \begin{array}{l}\text { This article cites } 39 \text { articles, } 15 \text { of which can be accessed free at: } \\ \text { http://rnajournal.cshlp.org/content/15/7/1251.full.html\#ref-list-1 }\end{array}\end{array}$

License

Email Alerting Receive free email alerts when new articles cite this article - sign up in the box at the Service top right corner of the article or click here.

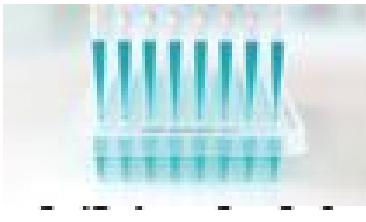

Providing Precise Solutions for your research.

To subscribe to RNA go to:

http://rnajournal.cshlp.org/subscriptions 